\title{
Raman spectra and phase behavior of the mixed solid N2-Ar at high pressure
}

Citation for published version (APA):

Kooi, M. E., \& Schouten, J. A. (1998). Raman spectra and phase behavior of the mixed solid N2-Ar at high pressure. Physical Review B: Condensed Matter, 57(17), 10407-10413.

https://doi.org/10.1103/PhysRevB.57.10407

DOI:

10.1103/PhysRevB.57.10407

Document status and date:

Published: 01/01/1998

\section{Document Version:}

Publisher's PDF, also known as Version of Record (includes final page, issue and volume numbers)

\section{Please check the document version of this publication:}

- A submitted manuscript is the version of the article upon submission and before peer-review. There can be important differences between the submitted version and the official published version of record. People interested in the research are advised to contact the author for the final version of the publication, or visit the $\mathrm{DOI}$ to the publisher's website.

- The final author version and the galley proof are versions of the publication after peer review.

- The final published version features the final layout of the paper including the volume, issue and page numbers.

Link to publication

\section{General rights}

Copyright and moral rights for the publications made accessible in the public portal are retained by the authors and/or other copyright owners and it is a condition of accessing publications that users recognise and abide by the legal requirements associated with these rights.

- Users may download and print one copy of any publication from the public portal for the purpose of private study or research.

- You may not further distribute the material or use it for any profit-making activity or commercial gain

- You may freely distribute the URL identifying the publication in the public portal.

If the publication is distributed under the terms of Article 25fa of the Dutch Copyright Act, indicated by the "Taverne" license above, please follow below link for the End User Agreement:

www.tue.nl/taverne

Take down policy

If you believe that this document breaches copyright please contact us at:

openaccess@tue.nl

providing details and we will investigate your claim. 


\title{
Raman spectra and phase behavior of the mixed solid $\mathrm{N}_{2}$-Ar at high pressure
}

\author{
M. E. Kooi and J. A. Schouten \\ Van der Waals-Zeeman Institute, University of Amsterdam, Valckenierstraat 65, 1018 XE Amsterdam, The Netherlands
}

(Received 6 October 1997; revised manuscript received 1 December 1997)

\begin{abstract}
The mixed solid $\mathrm{N}_{2}$-Ar has been studied for $x\left[\mathrm{~N}_{2}\right] \geqslant 0.75,130<T<470 \mathrm{~K}$, and $0<p \leqslant 20 \mathrm{GPa}$. A transition, very similar to the second order transition observed in pure $\delta-\mathrm{N}_{2}$, is still present in the mixed solid at $75 \mathrm{~mol} \%$ $\mathrm{N}_{2}$. An anomalous increase of the slope in a $p-T$ scan has been found, which is attributed to a redistribution of the Ar atoms near this transition. Although within the $\delta^{*}$ phase the Ar atoms are mainly located at the $a$ sites, the distribution of the Ar atoms about the sites depends on temperature and pressure. Also at these high pressures the Ar atoms are able to move through the lattice. The inhomogeneous broadening of the vibrational signal in the $\delta^{*}$ phase has been observed also at low temperatures. The phase diagram of the mixture has been studied. The $\delta$ - $\beta$ transition of pure $\mathrm{N}_{2}$ is shifted to lower temperatures in the mixture, while the $\beta$-fluid transition is shifted to higher temperatures. The results are in agreement with computer simulations. [S0163-1829(98)03017-3]
\end{abstract}

\section{INTRODUCTION}

The mixed solid $\mathrm{N}_{2}$-Ar constitutes a model system for studying the influence of a spherical molecule on the orientationally ordered and disordered phases of an anisotropic molecule. It is expected that the Ar atoms will dissolve substitutionally in the $\mathrm{N}_{2}$ crystal as the diameters of the molecules are nearly equal. Previously, the system $\mathrm{N}_{2}$-Ar has been studied extensively under its own vapor pressure as the phase diagram has some interesting features (see, e.g., Ref. 1). A high pressure study of the mixed solid at room temperature has been performed by Westerhoff and Feile for $x$ $\geqslant 0.59$, where $x$ is the mole fraction of nitrogen. ${ }^{2}$

It seems to be worthwhile to further investigate this interesting system under high pressure for the following reasons. At high pressure $(p>3 \mathrm{GPa})$ a phase transition occurs in pure nitrogen on cooling from the orientationally disordered hcp structure $\beta$ to a disordered cubic phase $\delta(P m 3 n)$ (see, e.g., Ref. 3). The unit cell of the $\delta$ phase contains eight molecules, two of which are positioned on the $a$ sites (the ones on the corners and the body-centered one) and they are spherically disordered. The other molecules are located in pairs at the faces of the unit cell ( $c$ sites) and they are orientationally disordered in a plane normal to the faces. An anomalous break in the slope of the vibrational Raman shift as a function of temperature along a quasi-isobar has been found by Scheerboom and Schouten. ${ }^{4}$ This break has been interpreted as a second order transition due to a localization of the orientations at low temperatures $\left(\delta_{\text {loc }}\right)$, which vanishes rapidly at the second order transition. Above the second order transition one speaks of $\delta_{\text {rot }}$. In accordance with the results of Ref. 4, Westerhoff et al. ${ }^{5}$ have found an anomaly in the pressure dependence of the linewidth of the $\nu_{1}$ vibron, which corresponds to the $a$ sites, at a pressure of $12 \mathrm{GPa}$ at room temperature.

This second order transition has also been detected using Monte Carlo simulations, ${ }^{6}$ by a change in the thermal expansion, the orientational order parameters, and the frequency. In this model the transition is caused by a localization of the orientations at low temperatures in combination with a change of preferential orientation of the disklike disordered $c$ sites. The orientational order parameter of the $\varphi$ coordinate of the $c$ sites, defined as $O_{d}^{\varphi}=\cos (4 \varphi)$, changes dramatically at the second order transition. The molecules at the spherelike disordered $a$ sites still rotate more or less freely in the $\delta_{\text {loc }}$ phase. Recently, also the orientational correlation's have been calculated, ${ }^{7}$ showing that neighboring disks are strongly correlated. Previously, the second order transition was studied by less sophisticated model calculations by Westerhoff and Feile. ${ }^{8}$ From their calculations, they expected a strong hindrance of the orientational degrees of freedom for the molecules on the spherelike disordered $a$ sites above $p$ $\approx 10 \mathrm{GPa}$ at room temperature.

Upon further cooling a first order phase transition occurs into an orthorhombic modification of the $\delta$ phase, the $\varepsilon$ phase $(R \overline{3} c)$ (see, e.g., Ref. 3), with a complete orientational order.

Westerhoff and Feile ${ }^{2}$ suggested a shift of the $\delta-\beta$ transition to higher pressures in $\mathrm{N}_{2}$-Ar for $x=0.59$. The high pressure spectra exhibit an asymmetry. They have measured an almost linear decrease of the intensity ratio of the $\nu_{1}$ and $\nu_{2}$ peak, corresponding to the $a$ and $c$ sites, respectively, as a function of concentration. For $x=0.59$, the two phases $\delta$ and $\beta$ coexist in a large pressure region.

Monte-Carlo simulations of Van Klaveren et al. ${ }^{9}$ on the orientational behavior of the mixed solid $\mathrm{N}_{2}-\mathrm{Ar}$ at high pressures predict a complex orientational ordering. In the model, the $\varepsilon^{*}-\delta^{*}$ (the asterisk denotes a mixed phase) transition shifts to lower temperatures, while the shift is larger for lower nitrogen contents. In the simulations the transition $\delta_{\text {rot }}-\delta_{\text {loc }}$ is still present and it is not shifted compared to pure nitrogen within the accuracy of the calculations. The transition can be characterized by the same order parameters as in pure nitrogen. The model predicts that the $\varepsilon$ phase is present down to $x=0.9$, but is most likely metastable. A phase, distinct from $\delta_{\text {loc }}$ and $\delta_{\text {rot }}$, might be present at low temperature. ${ }^{10}$

In this paper we report the investigation of the influence of argon on the second order transition $\delta_{\text {loc }}-\delta_{\text {rot }}$. In contrast to other investigations, ${ }^{2}$ this study has been carried out as a 
function of temperature along isobars, for reasons as will be explained. Moreover, the shift of the $\beta^{*}$-fluid and of the $\delta^{*}-\beta^{*}$ transition due to the addition of argon have been measured. The phase behavior in the region where computer simulations suggest the occurrence of another phase has been studied.

\section{EXPERIMENTAL METHOD}

The pressure was generated in a diamond-anvil cell (DAC) of the wedge type, ${ }^{11}$ connected to a temperature control system to study the phase behavior at various temperatures and pressures. The temperature stability was better than $0.1 \mathrm{~K}$. Research grade $\mathrm{N}_{2}$ and Ar with a purity better than 99.999\% have been mixed in a gas compressor and equilibrated for 14 days to allow for proper mixing. The temperature of the DAC is measured using copper-constantan thermocouples with an accuracy of about $0.5 \mathrm{~K}$. The $488.0 \mathrm{~nm}$ emission line of an argon ion laser is focused in the sample space. The laser focus can be visualized on a monitor, using a microscope. The signal was dispersed by a Dilor $X Y$ spectrometer and detected by a charge coupled device detector. The spectrograph has been calibrated before each measurement using an argon calibration lamp.

For the pressure measurement the ruby method was used with the scale of Mao et al. ${ }^{12}$ The temperature correction is taken from Vos and Schouten. ${ }^{13}$ To improve the accuracy of the pressure measurements the reference wavelength $R 1$ and $R 2$ at ambient pressure have been measured for each individual ruby chip. We made sure that the power of the laser was low enough during the pressure measurement to prevent heating of the ruby chip. For the Raman measurements the power of the laser was increased up to $200 \mathrm{~mW}$ and the laser focus is moved away from the ruby chip. The accuracy of the Raman measurements is about $0.2 \mathrm{~cm}^{-1}$ in the absolute wavenumber and the spectral width is about $0.75 \mathrm{~cm}^{-1}$.

In addition to the Raman measurements, the mixtures have been investigated by measuring the pressure in steps of about $2 \mathrm{~K}$ at nearly constant volume while heating or cooling the sample, a so-called $p-T$ scan. This quasi-isochoric scanning method can generally be used to detect phase transitions. The melting of the solid can also be observed through the microscope.

\section{RESULTS AND DISCUSSION}

In this work we will study the vibrational spectra and phase behavior of the mixed solid $\mathrm{N}_{2}-\mathrm{Ar}$, at the nitrogen-rich side of the phase diagram $\left(x\left[\mathrm{~N}_{2}\right] \geqslant 0.75\right)$, at pressures up to $20 \mathrm{GPa}$ in a diamond-anvil cell and temperatures from 130$470 \mathrm{~K}$. We have studied two compositions by Raman spectroscopy and $p-T$ scans, namely, 85 and $75 \mathrm{~mol} \% \mathrm{~N}_{2}$. In addition, the compositions 98.0 and $99.5 \mathrm{~mol} \%$ have been studied by $p-T$ scans only. We will discuss the results of the various parts of the phase diagram separately.

\section{A. The $\delta$ phase}

The Raman spectra of $\mathrm{N}_{2}$ in $\mathrm{N}_{2}$-Ar in the $\delta^{*}$ phase show two distinct peaks $\left(\nu_{1}\right.$ and $\left.\nu_{2}\right)$, corresponding to the $a$ and $c$ sites, respectively, as in pure $\mathrm{N}_{2}$. Figure 1 shows that in $\mathrm{N}_{2}$-Ar, the intensity of the $\nu_{1}$ peak has decreased compared

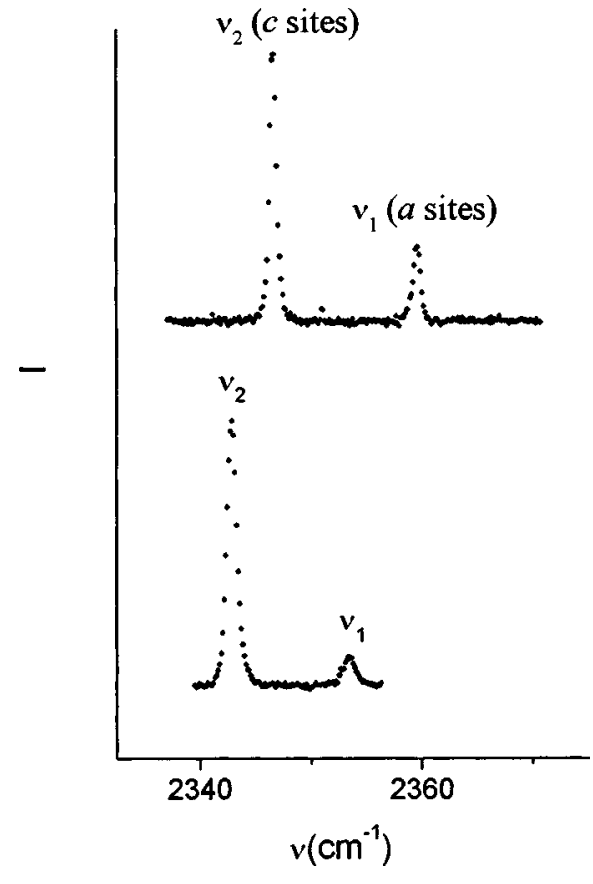

FIG. 1. Comparison between typical Raman spectra of pure $\mathrm{N}_{2}$ and $\mathrm{N}_{2}$ in $\mathrm{N}_{2}$-Ar, showing that the Ar atoms are mainly located at the $a$ sites. Upper spectrum, pure $\mathrm{N}_{2}, p=7.6 \mathrm{GPa}, T=156 \mathrm{~K}$, lower spectrum, $\mathrm{N}_{2}$-Ar, $x=0.85, p=7.1 \mathrm{GPa}$ and $T=195 \mathrm{~K}$.

to the that of the $\nu_{2}$ peak, in agreement with the results of Westerhoff and Feile at room temperature. ${ }^{2}$ These authors have explained the relative decrease of the intensity of the $\nu_{1}$ peak by stating that the Ar atoms dissolve in the $\delta^{*}$ phase and that they are positioned on the $a$ sites.

As two out of eight molecules of the unit cell of $\delta-\mathrm{N}_{2}$ are spherelike disordered ( $a$ sites), the 75 mol $\% \mathrm{~N}_{2}$ mixture has an interesting composition. At low temperatures the integrated intensity of the $\nu_{1}$ peak of $\mathrm{N}_{2}$ in $\mathrm{N}_{2}-\mathrm{Ar}, x=0.75$, is extremely low compared to the integrated intensity of the $\nu_{2}$ peak, but it is still present [Fig. 2(a)].

To study the temperature dependence of the intensity ratio of the two Raman peaks, we have performed the following experiment. Along a quasi-isobar, the temperature was first decreased till about $130 \mathrm{~K}$. Then the temperature was increased in steps of about $10 \mathrm{~K}$, and the two Raman peaks were carefully measured. The high-temperature spectrum [Fig. 2(a)] shows three peaks as it has been obtained in the $\delta^{*}-\beta^{*}$ coexistence region. Figure 2(a) also shows that the relative intensity of $\nu_{1}$ is higher at high temperature, but one can clearly observe that even at the $\delta^{*}-\beta^{*}$ transition temperature, the intensity ratio of $\nu_{1}$ to $\nu_{2}$ is still not 1:3 as in pure $\delta$ - $\mathrm{N}_{2}$.

It is useful to express the results in terms of the weighted integrated intensity ratio $W$ as defined by ${ }^{2}$

$$
W=x \frac{I\left(\nu_{1}\right)}{I\left(\nu_{1}\right)+I\left(\nu_{2}\right)},
$$

where $x$ denotes the mole fraction of $\mathrm{N}_{2}$, and $I\left(\nu_{1}\right)$ and $I\left(\nu_{2}\right)$ the integrated intensities of the two vibrational Raman modes. We have calculated $W$ as a function of temperature along quasi-isobars at 5.5, 7.0, and $11 \mathrm{GPa}$ in a $75 \mathrm{~mol} \% \mathrm{~N}_{2}$ mixture and at $6.7 \mathrm{GPa}$ in a $85 \mathrm{~mol} \% \mathrm{~N}_{2}$ mixture. First, the 

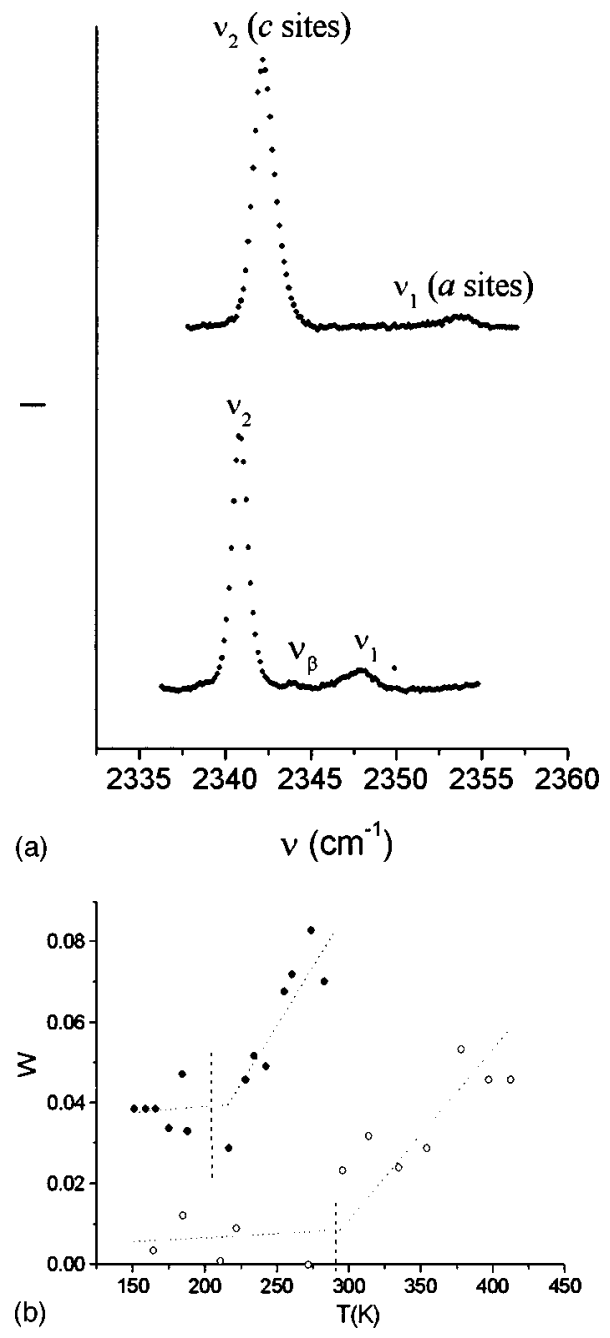

FIG. 2. (a) Typical Raman spectra of $\mathrm{N}_{2}$ in $\mathrm{N}_{2}-\mathrm{Ar}, x=0.75$ in the $\delta^{*}$ phase. Upper spectrum, $T=151 \mathrm{~K}$ and $p=6.4 \mathrm{GPa}$; lower spectrum, $T=283 \mathrm{~K}$ and $p=5.2 \mathrm{GPa}$. Note that the lower spectrum has been acquired at the $\delta^{*}-\beta^{*}$ transition. A small peak corresponding to $\nu_{\beta}$ is visible between $\nu_{1}$ and $\nu_{2}$. (b) Weighted integrated intensity ratio $W$ of the two Raman peaks of $\mathrm{N}_{2}$ in $\mathrm{N}_{2}$-Ar in the $\delta^{*}$ phase, $x=0.75$, showing that the relative intensity of the $\nu_{1}$ peak compared to the $\nu_{2}$ peak increases sharply as a function of temperature above second order transition in pure $\mathrm{N}_{2}$. Closed dots, $p \cong 5.5 \mathrm{GPa}$, open dots, $p \cong 11 \mathrm{GPa}$; dashed line, crossing point second order transition pure $\mathrm{N}_{2}$. Dashed and dotted lines are guides to the eyes.

integrated intensity ratio $W$ is nearly constant (one would expect a slight increase due to the thermal effects, but the accuracy is not high enough to be detected), but at higher temperatures it increases sharply [Fig. 2(b)]. In addition, $W$ is lower at higher pressure. In the $85 \mathrm{~mol} \%$ mixture, along the quasi-isobar of $6.7 \mathrm{GPa}$, we have measured an integrated intensity ratio of about 0.09 for $T<250 \mathrm{~K}$ and 0.13 at the $\delta^{*}-\beta^{*}$ transition.

This work shows that although the Ar atoms have a strong preference for the $a$ sites, the behavior is rather complex and depends on temperature and pressure. Since a small $\nu_{1}$ peak has been measured for $x=0.75$ in this work, these results suggest that for $x=0.75$ no superstructure with all the $\mathrm{Ar}$ atoms on the $a$ sites is formed. Westerhoff and Feile ${ }^{2}$ suggested that for $x=0.75$ ideally all $a$ positions are occupied by $\mathrm{Ar}$ and that the mixed system $\operatorname{Ar}_{0.25}\left(\mathrm{~N}_{2}\right)_{0.75}$ forms a superstructure. Since $W$ decreases as a function of pressure, it is concluded that the number of $\mathrm{Ar}$ atoms on the $a$ sites increases as a function of pressure along an isotherm. This is supported by computer simulations ${ }^{9}$ which have shown that the volume is lower if the Ar atoms are located on the $a$ sites than if they are located on the $c$ sites. As $W$ increases as a function of temperature along a quasi-isobar, it is concluded that the number of Ar atoms on the $c$ sites increases as a function of temperature. Even at these pressures ( $p$ $>6 \mathrm{GPa})$ the Ar atoms are able to move through the lattice. On the other hand, the molecules need to travel only a few lattice spacings in order to realize a redistribution. Although the intensity ratio increases as a function of temperature, at the $\delta-\beta$ transition temperature $W$ is still lower than the value for a random distribution of the Ar atoms over the $a$ and $c$ sites. Therefore, also at the $\delta-\beta$ coexistence region, the atoms still show a strong preference for the $a$ sites.

In agreement with Ref. 2 we have also found an asymmetry on the high-frequency site of $\nu_{2}$, for $x=0.85$, in the region $p \geqslant 13.8 \mathrm{GPa}$ and $T \leqslant 337 \mathrm{~K}$. The asymmetry gets more obvious at higher pressure. Due to the low intensity of the $\nu_{1}$ mode, it was impossible to investigate the line shape of this peak. Computer simulations on the mixed solid $\mathrm{N}_{2}-\mathrm{Ar}$ predict a splitting of the Raman frequencies $\nu_{2}$ in the $\delta_{\text {loc }}$ region. ${ }^{9}$ Therefore, this asymmetry might be associated with the splitting in the $\delta_{\text {loc }}$ region. However, at low pressures, there was no asymmetry in the $\delta_{\text {loc }}$ region within our experimental resolution. In contrast with Ref. 2 the simulations do not show a splitting of $\nu_{1}$.

Computer simulations at high pressure and for $x<0.9$, suggest a phase at low temperatures, distinct from the $\varepsilon$ phase. ${ }^{10}$ The lattice structure is that of the $\delta$ phase, but the preferential orientations of the molecules are different. It is still under investigation whether this phase is metastable or not. Therefore, we have studied the phase behavior for $x$ $=0.75$ at low temperatures by performing two $p-T$ scans in this region, at 10.3 and $13.5 \mathrm{GPa}$, respectively. In these scans, we have observed an abrupt change in the slope about $30 \mathrm{~K}$ above the $\varepsilon-\delta$ transition of pure $\mathrm{N}_{2}$ as shown in Fig. 3. A break in the slope in a $p-T$ scan generally corresponds to a change in the compressibility. Therefore, this break might be associated with this phase. The behavior of the $p-T$ scan is quite different from that at the $\varepsilon$ - $\delta$ transition in pure nitrogen. In the pure system one observes a discontinuous increase in pressure of about $0.07 \mathrm{GPa}$ and an increase of the Raman frequency $\nu_{2}$ of about $0.2 \mathrm{~cm}^{-1}$ at the $\varepsilon-\delta$ transition along a quasi-isochore. ${ }^{14}$ No anomalous behavior of the Raman frequency $\nu_{2}$ could be detected in this region. We were unable to measure $\nu_{1}$ in this region as at these low temperatures the peak intensity is extremely low.

\section{B. Second order transition}

To study the occurrence of a second order transition it is convenient to have the data on a clearly defined thermodynamic path. At high densities the pressure is a measure for the average forces on the molecules, while by a change in orientation the axial components of these forces will change as described in Refs. 15 and 4. The shift of the Raman frequency due to the surrounding medium is mainly caused by 


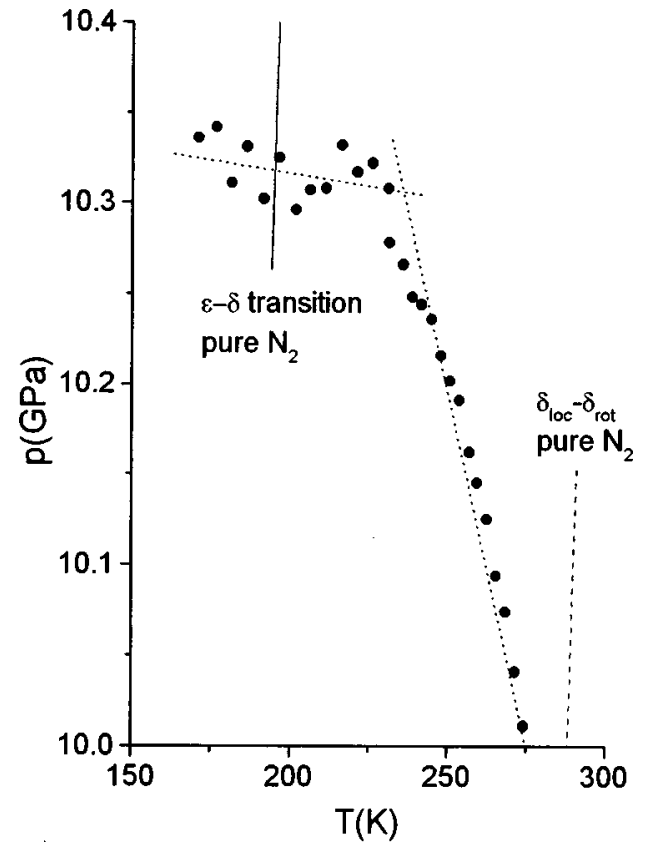

FIG. 3. Typical $p-T$ scan (๑), $x=0.75$, showing an abrupt change in slope about $30 \mathrm{~K}$ above the $\varepsilon-\delta$ transition of pure $\mathrm{N}_{2}$. Dotted lines are guides to the eyes.

the axial components of the intermolecular forces on the molecule. Therefore, measuring the data along isobars is a suitable method to study the frequency change due to a change in orientation, because the average forces hardly change. Due to the experimental technique the data have been obtained along quasi-isobars. We have corrected these data to isobars by plotting the Raman frequencies, $\nu_{1}$ and $\nu_{2}$, against pressure at constant temperature. These two curves can be fitted by linear equations within experimental accuracy. By this procedure we have determined the pressure dependence of the Raman frequencies at different temperatures. The slopes of these fits have been plotted as a function of temperature, and the slopes can be fitted with polynomial functions of the second order, which gives the change in frequency due to a pressure change as a function of temperature. This function has been used to correct the individual data points of $\nu$-T scans to the same pressure. We have corrected the points to the average pressure during that particular scan. Note that by this procedure we do not smooth the data and we do not change essentially the temperature behavior of $\nu$.

A typical plot of the Raman frequencies $\nu_{1}$ and $\nu_{2}$, as a function of temperature along an isobar has been given in Fig. 4. Since in the $\mathrm{N}_{2}$-Ar mixture at higher temperature, not all the argon atoms are located at the $a$ sites, we were able to measure both $\nu_{1}$ and $\nu_{2}$, for $x=0.75$ as well as the $x$ $=0.85$. The experiments show a break in the slope along an isobar. The effect has been measured in the $85 \mathrm{~mol} \%$ mixture at 5.4, 6.2, and 6.7 GPa. It is not clear if the break is still present at the isobar $p=14.4 \mathrm{GPa}$. The $75 \mathrm{~mol} \%$ mixture has been studied at 5.5, 7.0, and 8.5 GPa. Again in the highpressure isobar, $p=11 \mathrm{GPa}$, the effect is less obvious. Since the intensity of the $\nu_{1}$ mode is reduced in the mixed solid, it is not possible to determine the temperature of the break in the slope very accurately. For the $x=0.75$ we estimate the

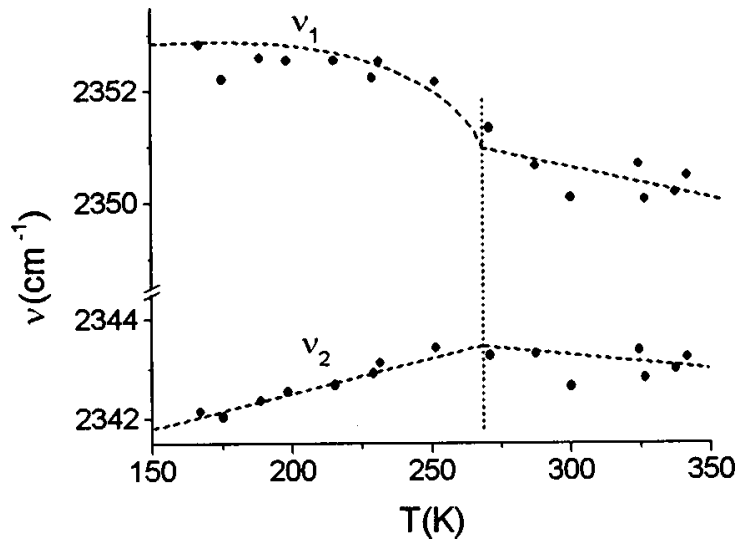

FIG. 4. $\nu_{1}$ and $\nu_{2}$ as a function of temperature in $\mathrm{N}_{2}$ - $\mathrm{Ar}, x$ $=0.85$, corrected to the isobar $p=6.2 \mathrm{GPa}$, showing a break in the slope at the second order transition. Dashed and dotted lines are guides to the eyes.

temperature of the break to be 0 to $30 \mathrm{~K}$ above the second order transition temperature of pure $\mathrm{N}_{2} \cdot{ }^{4}$ No additional Raman peaks have been observed near the break. Since the frequencies have been measured in temperature steps of about $10 \mathrm{~K}$ and considering the accuracy, a small hysteresis of a few K cannot be excluded.

In contrast to the behavior in pure nitrogen, the slope of the pressure versus temperature in a $p-T$ scan increases strongly near the temperature of the break in a small temperature range for both the 85 and $75 \mathrm{~mol} \%$ mixture (Fig. 5). There is no discrepancy between heating and cooling runs, so there seems to be no metastability or hysteresis. The dotted line represents a fit through the end points of the steep slopes of six different scans.

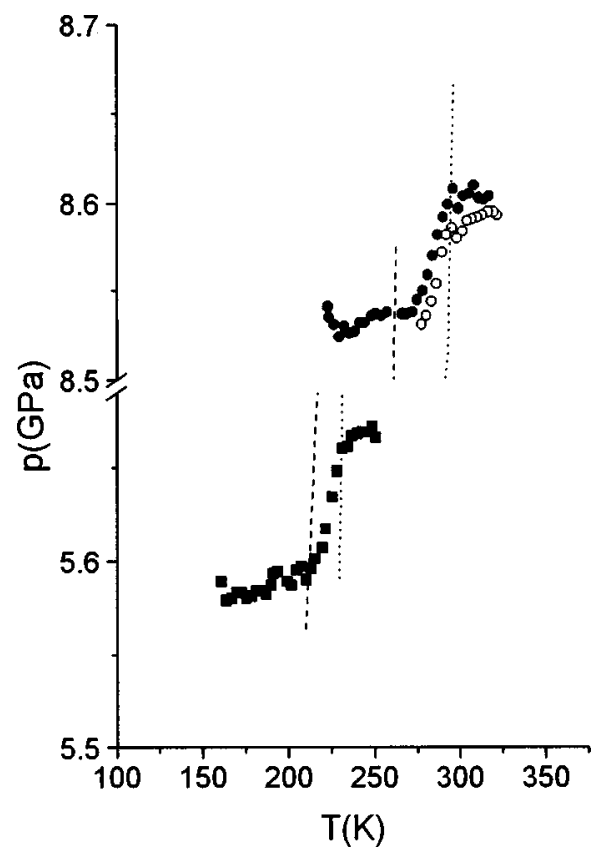

FIG. 5. Typical heating and cooling $p-T$ scans, $x=0.75$, near the second order transition of pure $\mathrm{N}_{2}$. Closed symbols, heating scans; open symbols, cooling scan; dotted line, fit through end points of steep slopes in $p-T$ scans; dashed line, second order transition pure $\mathrm{N}_{2}$ (Ref. 4). 


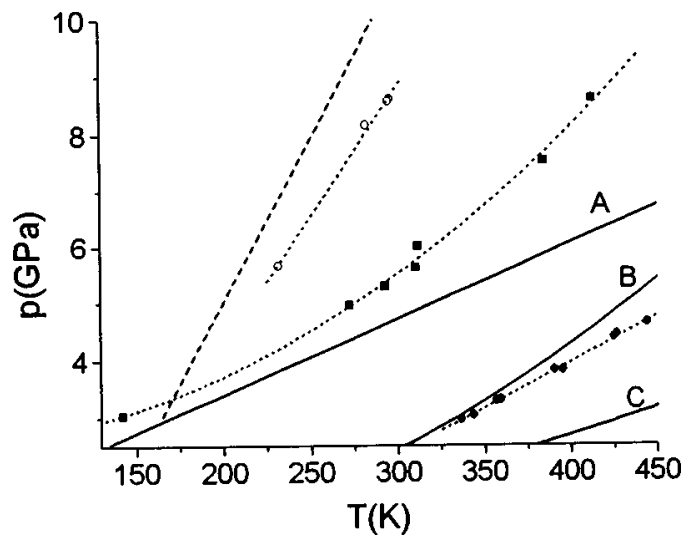

FIG. 6. $p$ - $T$ phase diagram. Solid lines: (a) $\delta-\beta$ pure $\mathrm{N}_{2}$ (Ref. 16), (b) melting line pure $\mathrm{N}_{2}$ (Ref. 16), (c) melting line pure $\mathrm{Ar}$ (Ref. 19), dashed line, $\delta_{\text {loc }} \delta_{\text {rot }}($ Ref. 4$)$; this work $(x=0.75)$ : solid squares, $\delta^{*}-\beta^{*}$; solid circles, $\beta^{*}$-fluid; open circles, end points of sharp $p-T$ slope near $\delta_{\text {loc }}-\delta_{\text {rot }}$. Note that for the sake of clarity only data points of $x=0.75$ have been plotted. Dotted lines are guides to the eyes.

Generally, in a binary system, a three-phase line is followed in a range of a few $\mathrm{K}$ during a $p-T$ scan, as this line is uniquely defined. Therefore, at a three-phase line the slopes at the pressure jumps in different $p-T$ scans should be in line. As one can see in Fig. 5, this is clearly not the case with the peculiar $p-T$ behavior of the mixed solid near the break. The character of the pressure jumps could correspond to the $p-T$ behavior in a small two phase coexistence region. However, a two-phase region does not seem to be very likely according to the following arguments. In the case of a twophase region one would expect to measure the vibrational modes of both the low-temperature and the high-temperature phase, but we did not find any additional Raman peaks, neither a discontinuity of the Raman frequencies near the region in which the peculiar $p-T$ jump occurs. Further, no metastability or hysteresis between heating and cooling runs has been observed, which would be expected in the case of a two-phase region.

It should be noted that in the mixed solid the presence of argon atoms gives extra possibilities for a contribution to the entropy. On the other hand, computer simulations ${ }^{9}$ have shown that the volume of the system is larger when the argon atoms are located on the $c$ sites than if they are located on the $a$ sites. Therefore, this increase of the pressure might be caused by a rearrangement of the Ar atoms about both the $a$ and $c$ sites. This should result in an increase of the intensity of $\nu_{1}$ and as one can see in Fig. 2(b) the intensity ratio of $\nu_{1}$ and $\nu_{2}$ indeed increases strongly at temperatures above the second order transition of pure nitrogen.

In pure nitrogen the frequency of the upper mode, $\nu_{1}$ changes rapidly near the second order transition as a function of temperature along an isobar, while the frequency of the lower mode, $\nu_{2}$, changes only slightly. ${ }^{4}$ The behavior of the calculated frequencies resembles the experimental behavior. ${ }^{6}$ Although, in the model hardly any change in the orientational behavior of the molecules at the $a$ sites has been observed, the calculated vibrational frequency behavior of these sites changes at the second order transition, due to the change in orientational behavior of the twelve molecules at

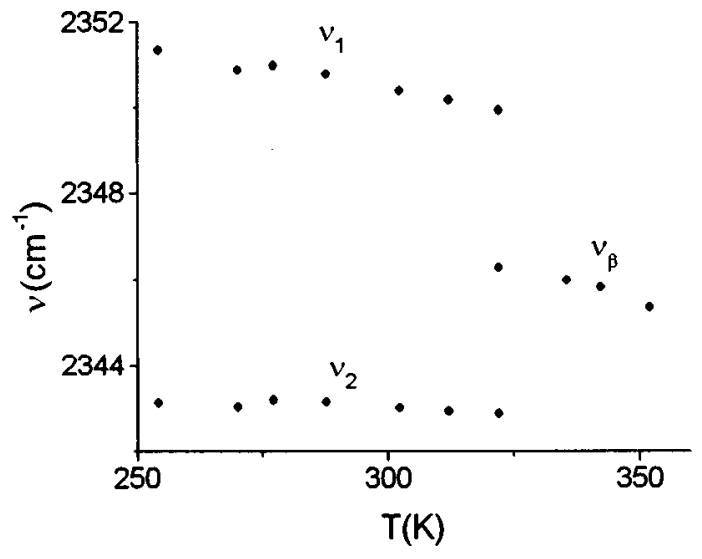

FIG. 7. Typical $\nu-T$ scan near the $\delta^{*}-\beta^{*}$ transition, $x=0.75$, $p \approx 6 \mathrm{GPa}$. In the $\delta^{*}$ phase one can distinct two vibrational Raman peaks corresponding to the $a$ and $c$ sites, respectively, while in the $\beta^{*}$-phase there is only one vibrational Raman peak.

the nearest neighbor (disklike disordered) $c$ sites. The anomaly in the linewidth of the $\nu_{1}$ vibron near the second order transition, as reported by Westerhoff et al., ${ }^{5}$ could also be caused by a change in the orientational behavior of the neighboring disklike disordered $c$ sites.

In the mixture the experimental behavior of the Raman frequencies as a function of temperature along an isobar, resembles that in pure nitrogen at the second order transition. In addition, the behavior of the calculated Raman frequencies of $\mathrm{N}_{2}$ in $\mathrm{N}_{2}-\mathrm{Ar}$ in computer simulations of Van Klaveren et al. ${ }^{9}$ resembles the experimental behavior in the mixture. The break in the frequencies as a function of temperature is caused by a freezing of the orientations of the molecules on the $c$ sites, as in simulations on pure nitrogen. ${ }^{6}$ The resemblance with the pure system and the agreement between experiments and computer simulations on the mixture, suggests that a similar transition to the second order transition in pure nitrogen is present in the mixture. A small discontinuity of the Raman frequencies, disregarding a continuous transition, cannot be excluded. The fact that the break in the slope of the frequencies versus the temperature is still present for $x$ $=0.75$, when most of the $a$ sites are occupied by Ar atoms, supports the idea that the transition is caused by a freezing of the orientations of the molecules on the $c$ sites.

\section{The $\delta-\beta$ transition}

The $\delta^{*}-\beta^{*}$ transition of the mixture can be clearly observed by Raman spectroscopy. At the transition temperature, we observe three peaks. In addition, the transition can be clearly detected by $p-T$ scans, since at the phase transition one observes a discontinuity of the pressure of about 0.2 $\mathrm{GPa}$, comparable with that in pure $\mathrm{N}_{2} .{ }^{16}$ We have performed $p$ - $T$ scans in mixtures with 99.5, 98.0, 85, and $75 \mathrm{~mol} \% \mathrm{~N}_{2}$ at several pressures and $\nu$-T scans in mixtures with 85 and $75 \mathrm{~mol} \% \mathrm{~N}_{2}$. The two phase coexistence line for $x=0.75$ has been plotted in a $p-T$ diagram (Fig. 6). The coexistence lines for the other compositions are between those of $x$ $=0.75$ and of $x=1$. One can observe that the transition is shifted to lower temperatures as a function of concentration. This corresponds to a shift to higher pressures at the $300 \mathrm{~K}$ isotherm as found by Westerhoff and Feile. ${ }^{2}$ For $x=0.75$, the 


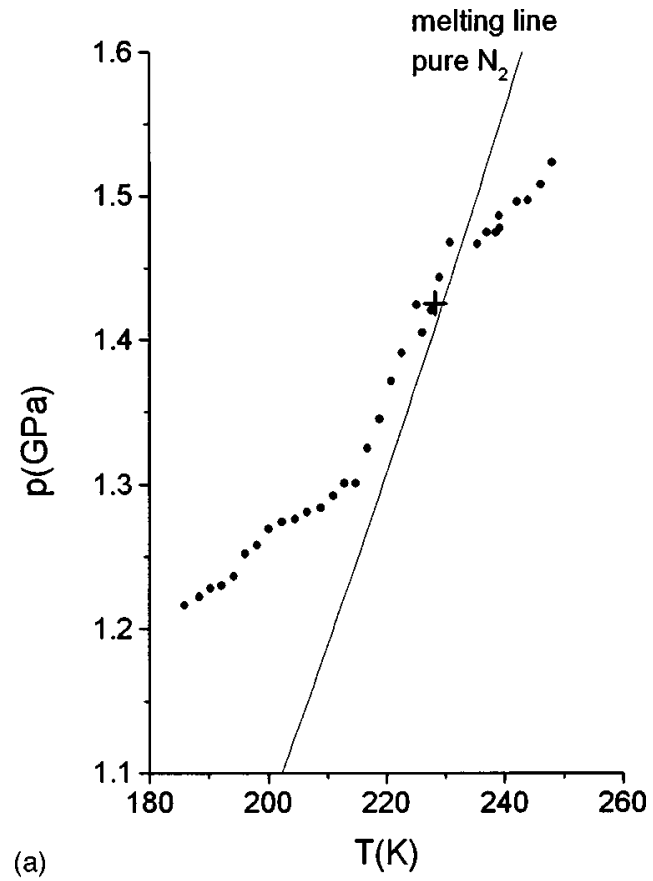

(a)

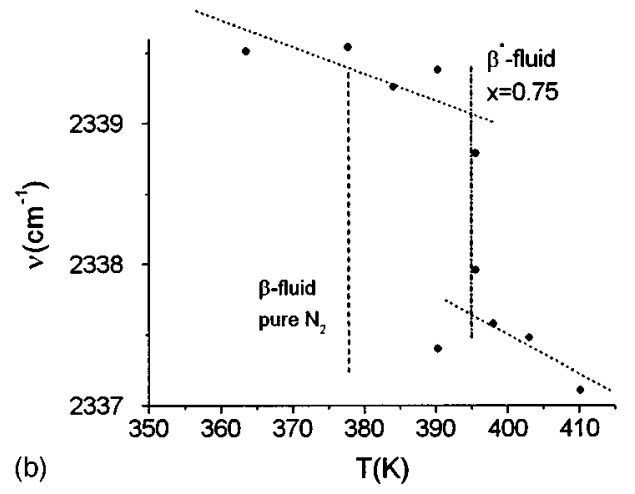

FIG. 8. (a) Typical $p$ - $T$ scan (O) near the $\beta^{*}$-fluid transition, $x=0.85$; cross, visual observation of melting point. (b) $\nu-T$ scan near the $\beta^{*}$-fluid transition, $x=0.75, p \cong 3.8 \mathrm{GPa}$. Dotted line is a guide to the eyes.

shift is about $40 \mathrm{~K}$ at $5 \mathrm{GPa}$, while the shift increases as a function of pressure. In a heating run, the slope of the pressure versus the temperature increases sharply in the $\delta^{*}-\beta^{*}$ coexistence region, which is followed in a range of about 5 $\mathrm{K}$. In a cooling run, the pressure first drops discontinuously due to metastability and subsequently the same coexistence region as in a heating run is followed in a range of about 1-2 $\mathrm{K}$. Therefore, we can conclude that the system shows no hysteresis at this transition. One can observe that for $x$ $=0.98$, the $\delta^{*}-\beta^{*}$ transition is already shifted to about $8 \mathrm{~K}$ below the transition line in pure $\mathrm{N}_{2}$. The character of the $p-T$ scans for $x=0.75$, suggest a small $\delta^{*}-\beta^{*}$ two-phase region, in agreement with expectations. In Fig. 7 a typical $\nu$ - $T$ scan at $p \approx 6 \mathrm{GPa}$ and $x=0.75$, has been given. In the $\delta^{*}$ phase one can distinguish two vibrational Raman peaks corresponding to the $a$ and $c$ sites, while in the $\beta^{*}$ phase there is only one peak. In the coexistence region three peaks have been observed, corresponding to the two modes of the $\delta^{*}$ phase and the mode of the $\beta^{*}$ phase.

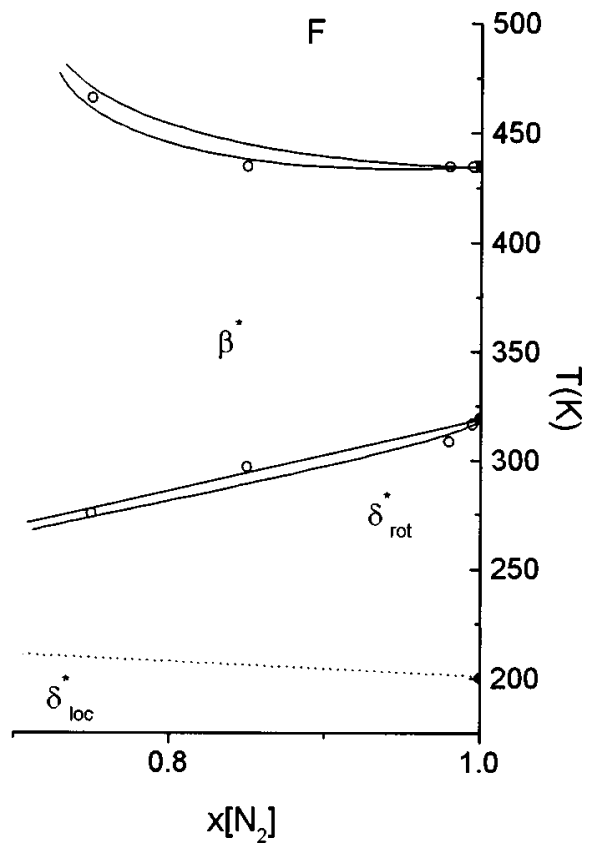

FIG. 9. Proposed $T-x$ phase diagram at $5 \mathrm{GPa}$. Two phase regions are enlarged for the sake of clarity.

\section{The $\beta$-fluid transition}

We have measured the shift of the $\beta$-fluid transition by $p$ - $T$ scans in mixtures with 99.5, 98.0, 85 and $75 \mathrm{~mol} \% \mathrm{~N}_{2}$ respectively at several pressures, using the discontinuity in the pressure. Further, we have measured the transition line by $\nu$ - $T$ scans in the 85 and $75 \mathrm{~mol} \%$ mixture, using the discontinuity in the Raman frequency. The melting of the solid has been observed through the microscope for the 85 mol \% mixture. The $p-T, \nu-T$ scans, and the visual observations are in mutual agreement. For $x \geqslant 0.85$ the transition has not shifted within the experimental accuracy [Fig. 8(a)]. The deviation with pure nitrogen is within experimental accuracy. For $x=0.75$ the transition line has shifted to higher temperatures given the pressure (Fig. 6). At $3 \mathrm{GPa}$ the transition is shifted to about $15 \mathrm{~K}$ above the melting line of pure $\mathrm{N}_{2} \cdot{ }^{16}$ Note that at ambient pressure ${ }^{17,18}$ the shifts are only noticeable for $x<0.6$, while at higher pressure 0.75 is more than sufficient. Considering the higher melting temperature of $\operatorname{argon}^{19}$ it is expected that the $\beta$-fluid transition will shift to higher temperatures at increasing argon concentrations. Typical $p-T$ and $\nu$-T scans have been plotted in Figs. 8(a) and $8(\mathrm{~b})$. In Fig. 8(a) one can observe that the discontinuity of the pressure at the $\beta$-fluid transition is about $0.15 \mathrm{GPa}$, comparable to pure $\mathrm{N}_{2} .{ }^{16}$ Figure 8 (b) shows that, as usual, the frequency is higher in the solid phase. In the range of a few $\mathrm{K}$ the two phases coexist and two Raman peaks have been observed simultaneously.

As the molecular diameters of $\mathrm{Ar}$ and $\mathrm{N}_{2}$ are nearly equal, and because the Ar atoms dissolve into the $\delta^{*}$ phase as shown above, we expect that the Ar atoms will also dissolve into the $\beta$ phase. Since at $x=0.75$ the melting line has shifted towards higher temperatures, a three-phase line should have been measured in the $p-T$ and $\nu$-T scans in case argon did not dissolve into $\beta-\mathrm{N}_{2}$. Such behavior has not been found, and thus argon dissolves into $\beta-\mathrm{N}_{2}$. Figure 8(a) 
shows that the solubility is almost complete, because in the case of a large two-phase region the discontinuity in slope would have been much smaller than in pure nitrogen.

Figure 9 shows the proposed $T-x$ phase diagram for molar fractions greater than 0.75 , at $p=5 \mathrm{GPa}$. The open circles are interextrapolated or extrapolated results of the phase lines described above.

\section{CONCLUSIONS}

The present measurements demonstrate that, in the mixed solid $\mathrm{N}_{2}-\mathrm{Ar}$, the Ar atoms dissolve into the $\beta$ and $\delta$ phase of $\mathrm{N}_{2}$. Within the $\delta^{*}$ phase the Ar atoms are mainly located at the $a$ sites, but the preference of the Ar atoms for the $a$ sites decreases as a function of temperature and increases as a function of pressure. It is shown that, at these high pressures, the Ar atoms are able to move through the lattice. A similar transition as the second order transition observed in pure nitrogen is still present in the mixed solid for $x=0.75$. Our results are in agreement with computer simulations in the $\delta^{*}$ phase. An anomalous increase of the slope in a $p-T$ scan has been found, which is attributed to a redistribution of the $\mathrm{Ar}$ atoms near the second order transition. The inhomogeneous line broadening due to the addition of Ar, as found by Westerhoff and Feile, ${ }^{2}$ has been confirmed. Given the pressure, the $\delta^{*}-\beta^{*}$ transition shifts to lower temperatures due to the addition of argon. The $\beta^{*}$-fluid transition shifts to higher temperatures at decreasing nitrogen concentrations given the pressure. In contrast to the ambient pressure results, at high pressure the shift is already noticeable at nitrogen concentrations smaller than 75 mol \%.
${ }^{1}$ J. A. Hamida, N. S. Sullivan, and M. D. Evans, Phys. Rev. Lett. 73, 2720 (1994).

${ }^{2}$ T. Westerhoff and R. Feile, Phys. Rev. B 54, 913 (1996).

${ }^{3}$ R. L. Mills, B. Olinger, and D. T. Cromer, J. Chem. Phys. 84, 2837 (1986).

${ }^{4}$ M. I. M. Scheerboom and J. A. Schouten, Phys. Rev. Lett. 71, 2252 (1993).

${ }^{5}$ T. Westerhoff, A. Wittig, and R. Feile, Phys. Rev. B 54, 14 (1996).

${ }^{6}$ A. Mulder, J. P. J. Michels, and J. A. Schouten, Phys. Rev. B 57, 7571 (1998).

${ }^{7}$ J. A. Schouten and A. Mulder, J. Low. Temp. Phys. (to be published May 1998).

${ }^{8}$ T. Westerhoff and R. Feile, Z. Phys. B 100, 417 (1996).

${ }^{9}$ E. P. van Klaveren, J. P. J. Michels, and J. A. Schouten, J. Low Temp. Phys. (to be published May 1998).
${ }^{10}$ E. P. van Klaveren (private communication).

${ }^{11}$ H. Wieldraaijer, J. A. Schouten, and N. J. Trappeniers, High Temp.-High Press. 15, 87 (1983).

${ }^{12}$ H. K. Mao, J. Xu, and P. M. Bell, J. Geophys. Res. 91, 4673 (1986).

${ }^{13}$ W. L. Vos and J. A. Schouten, J. Appl. Phys. 69, 6744 (1991).

${ }^{14}$ M. I. M. Scheerboom and J. A. Schouten, J. Phys.: Condens. Matter 3, 8305 (1991).

${ }^{15}$ J. P. J. Michels, M. E. Kooi, and J. A. Schouten, J. Chem. Phys. 108, 2695 (1998).

${ }^{16}$ W. L. Vos and J. A. Schouten, J. Chem. Phys. 91, 6302 (1989).

${ }^{17}$ C. S. Barret and L. Meyer, J. Chem. Phys. 42, 107 (1965).

${ }^{18}$ L. D. Yantesevich, A. I. Prokhvatilov, I. N. Krupskii, and A. S. Baryl'nik, Sov. J. Low Temp. Phys. 12, 170 (1986).

${ }^{19}$ L. van der Putten and J. A. Schouten, High Temp.-High Press. 18, 393 (1986). 